\title{
Assessment of Variation Throughout the Year in the Incidence of Idiopathic Sudden Sensorineural Hearing Loss
}

\author{
*Deya N. Jourdy, *Luke A. Donatelli, †Jonathan D. Victor, \\ and $* \dagger+$ Samuel H. Selesnick \\ Departments of *Otorhinolaryngology, $\dagger$ Neurology and Neuroscience, and $\$$ Neurosurgery, Weill Cornell \\ Medical College, New York, New York, U.S.A.
}

Objective: This study was conducted to determine whether the incidence of idiopathic sudden sensorineural hearing loss (ISSHL) varies throughout the year.

Study Design: This study is a retrospective case review.

Setting: This study was conducted at a tertiary referral center within a teaching hospital.

Patients: Inclusion criteria were a diagnosis of ISSHL confirmed by audiometric data. Exclusion criteria were intracranial neoplasms, a history of Ménière's disease, previous ear procedures, chemotherapy, or radiation therapy to the head or neck. There was no exclusion criterion based on age. Ninetyseven patients met these criteria. The median age was 52 years (range, 26-85 yr), and there were $53(54.6 \%)$ women and 44 (45.4\%) men.

Main Outcome Measures: Monthly incidence counts were compiled across a 3-year period. Counts were analyzed for uneven incidence distributions and seasonal variation via standard statistical tests.

Results: Overall, no evidence was found for an uneven distribution or for a peak either by $\chi^{2}(p>0.1)$, which assesses for any uneven distribution, or by the circular mean $(p>0.1)$, which assesses for a pattern of seasonal variation. In the subset of patients ( 24 of $97 ; 24.7 \%$ ) who reported experiencing an upper respiratory infection before or concurrent with the onset of ISSHL, no evidence was found for an uneven distribution of hearing loss onset throughout the year either by $\chi^{2}(p>0.1)$ or by the circular mean $(p>0.1)$.

Conclusion: The results of this study suggest that ISSHL incidence does not display uneven distribution throughout the year. Key Words: Incidence-Seasonal variation-Sensorineural hearing loss-Sudden hearing loss.

Otol Neurotol 31:53-57, 2010.
Idiopathic sudden sensorineural hearing loss (ISSHL) occurs in an estimated 5 to 20 per 100,000 persons per year (1). A number of hypotheses have been proposed to explain ISSHL, including vascular occlusion, breaks in labyrinthine membranes, and viral infection (2). The viral hypothesis has been supported by clinical observations that hearing loss is associated with infectious diseases (e.g., herpesvirus, cytomegalovirus, and human immunodeficiency virus) (3), and that ISSHL frequently is preceded by an upper respiratory infection (URI) (2).

Because many viruses display seasonal patterns of incidence, one method of evaluating the association between viral infection and ISSHL is to determine whether

Address correspondence and reprint requests to Samuel H. Selesnick, M.D., F.A.C.S., Department of Otorhinolaryngology, Weill Cornell Medical College, 1305 York Avenue, 5th Floor, New York, NY 10021; E-mail: Shselesn@med.cornell.edu variations in the incidence of ISSHL follow a pattern similar to that of a seasonal viral infection. Therefore, we conducted a retrospective chart review of patients with ISSHL seen at our department during a 3-year period.

\section{MATERIALS AND METHODS}

From May 1, 2005 to April 30, 2008, 131 patients were given a new diagnosis of "unspecified sudden hearing loss" (International Classification of Diseases -9th Revision-Clinical Modification code 388.2) at the Department of Otorhinolaryngology at the Weill Cornell Medical College, which is a tertiary care center in New York, NY.

All research was conducted with the approval of the New York Presbyterian Hospital-Weill Cornell Medical College Institutional Review Board. Charts were evaluated according to the following inclusion criteria: 1) the reviewer was able to confirm the diagnosis of ISSHL based on audiometric data; 
2) the month and year of onset of hearing loss were reported by the examining physician or audiologist; and 3) audiograms reported air- and bone-conduction values at $0.25,0.50,1.0$, $2.0,3.0,4.0,6.0$, and $8.0 \mathrm{kHz}$. Charts with audiograms indicating a sensorineural (i.e., air and bone conduction) hearing loss of greater than or equal to $20 \mathrm{~dB}$ from a baseline of $0 \mathrm{~dB}$ averaged over 3 contiguous audiometric frequencies, within 3 or fewer days, were included for further review. Among these charts, we identified the subset in which audiograms indicated a hearing loss of greater than or equal to $30 \mathrm{~dB}$ from a baseline of $0 \mathrm{~dB}$ averaged over 3 contiguous audiometric frequencies. A threshold for hearing loss of greater than or equal to $30 \mathrm{~dB}$ is a widely accepted definition for ISSHL that has been adopted by the National Institute on Deafness and Other Communication Disorders (NIDCD) (4), whereas the threshold of greater than or equal to $20 \mathrm{~dB}$ has been used by some investigators as a more inclusive criterion (3).

Although charts were initially selected based on the date of the office visit on which hearing loss was diagnosed, the incidence of ISSHL among the study population was determined based on the month and year in which the patient reported experiencing sudden hearing loss. If the month and year of onset of sudden hearing loss were not recorded, or if the onset of sudden hearing loss did not occur within the 36-month study period, the chart was excluded. The date of the office visit on which a patient was diagnosed with ISSHL often did not match the reported date of onset of hearing loss. Both the date of the office visit and the date of hearing loss were recorded, and median and range values for the time between hearing loss onset and the diagnosis of ISSHL at our institution are presented in the Results section in the succeeding paragraphs.

Patients with concurrent intracranial neoplasms or a history of Ménière's disease, previous ear procedures, chemotherapy, or radiation therapy to the head or neck were excluded from the study. There was no exclusion criterion based on age, but all of the patients included in the study were adults. After applying the previously discussed inclusion and exclusion criteria to the 131 patients who were given a new diagnosis of "unspecified sudden hearing loss" during the study period, the study population included 97 patients, among whom there were $53(54.6 \%)$ women and $44(45.4 \%)$ men. There was a median age of 52 years (range, 26-85 yr). Within this population, $84(86.6 \%)$ patients experienced hearing loss of greater than or equal to $30 \mathrm{~dB}$. Among these patients, the median age was 52 years (range, 26-85 yr), and there were 45 (53.6\%) women and $39(46.4 \%)$ men.

Incidence values for the onset of ISSHL were compiled for each of the 36 months during the study period. The incidence values were then totaled for each 12-month period (May to April), and a $\chi^{2}$ test was performed on the annual incidence values to determine if there was a significant difference in inci- dence across the 3 years of the study period. Monthly ISSHL incidence values were then analyzed within each 12-month period (May to April) to determine whether there was evidence for uneven distribution of ISSHL incidence over the course of each of the 3 individual 12-month periods of the study. In addition, incidence values were grouped by month across all 3 years of the study (e.g., incidence values from May 2005, May 2006, and May 2007 were summed) to yield a total incidence value for each month of the year, and these data were also analyzed for evidence of uneven distribution of ISSHL. To determine whether there was a significant variation of incidence within each individual 12-month period and across all 3 years of the study, 2 tests were performed: the $\chi^{2}$ test, which assesses for any deviation from uniform incidence, and the circular mean, which specifically assesses for seasonality (i.e., a sinusoidal variation in incidence during the course of the year). These tests are complementary: the circular mean test is more sensitive for detecting seasonality (5) but may miss other kinds of variation throughout the year such as 1 month with an anomalously high incidence. Significance levels of $\chi^{2}$ and circular mean statistics were determined by standard formulas for large sample sizes ( $\mathrm{n} \geq 20$ for $\chi^{2} ; \mathrm{n}>8$ for circular mean) and to screen smaller sample analyses for significance (6). For smaller sample sizes, potentially significant $p$ values were corroborated by Monte Carlo methods and performed by Matlab scripts written by the third author.

Charts were also reviewed to identify pertinent associated history elicited from all patients included in the study such as tinnitus, dizziness, aural pressure or fullness, otalgia, history of hearing loss, familial history of hearing loss, and history of noise exposure. Furthermore, charts were reviewed for history that may be pertinent to the clinical picture of the patient presenting with ISSHL but were not uniformly evaluated in all patients such as symptoms of URI preceding or concurrent with the onset of sudden hearing loss.

\section{RESULTS}

We separately analyzed all of the patients with a hearing loss of greater than or equal to $20 \mathrm{~dB}$ and the subset of patients who experienced hearing loss of greater than or equal to $30 \mathrm{~dB}$. Most findings were comparable between these 2 groups. Within the whole population with greater than or equal to $20 \mathrm{~dB}$ hearing loss (Table 1), the average total monthly incidence of hearing loss was 2.7 incidents per month (range, $0-7$ ), and there was a median of 7 days (range, $0-458 \mathrm{~d}$ ) between the onset of hearing loss and the diagnosis of ISSHL. Annual ISSHL

TABLE 1. Monthly incidence values for the reported onset of ISSHL for patients with hearing loss of $\geq 20 \mathrm{~dB}$

\begin{tabular}{|c|c|c|c|c|c|c|c|c|c|c|c|c|c|}
\hline & May & Jun & Jul & Aug & Sep & Oct & Nov & Dec & Jan & Feb & Mar & Apr & Total \\
\hline 2005 to 2006 & 1 & 2 & 2 & 1 & 3 & 3 & 2 & 0 & 2 & 3 & 4 & 1 & 24 \\
\hline 2006 to 2007 & 1 & 4 & 2 & 3 & 4 & 4 & 3 & 2 & 4 & 2 & 7 & 5 & 41 \\
\hline 2007 to 2008 & 5 & 6 & 1 & 3 & 0 & 4 & 3 & 1 & 2 & 1 & 2 & 4 & 32 \\
\hline Total & 7 & 12 & 5 & 7 & 7 & 11 & 8 & 3 & 8 & 6 & 13 & 10 & 97 \\
\hline
\end{tabular}

Annual ISSHL incidence did not vary significantly across the 3 years of the study $\left(\chi^{2}, p>0.1\right)$.

No evidence of uneven distribution throughout the year with data pooled by month (bottom row) either by $\chi^{2}$ or by the circular mean ( $p>0.1$ for both).

No evidence of uneven distribution when the years were analyzed individually (each of the first 3 rows) by $\chi^{2}$ or by the circular mean ( $p>0.1$ for both for all years). 
TABLE 2. Monthly incidence values for the reported onset of ISSHL for patients with hearing loss of $\geq 20 \mathrm{~dB}$ who reported concurrent or preceding URI

\begin{tabular}{|c|c|c|c|c|c|c|c|c|c|c|c|c|c|}
\hline & May & Jun & Jul & Aug & Sep & Oct & Nov & Dec & Jan & Feb & Mar & Apr & Total \\
\hline 2005 to 2006 & 1 & 0 & 0 & 0 & 1 & 1 & 2 & 0 & 1 & 1 & 0 & 0 & 7 \\
\hline 2006 to 2007 & 1 & 0 & 0 & 0 & 0 & 1 & 0 & 0 & 2 & 1 & 2 & 1 & 8 \\
\hline 2007 to 2008 & 0 & 2 & 1 & 0 & 0 & 1 & 1 & 0 & 1 & 0 & 1 & 2 & 9 \\
\hline Total & 2 & 2 & 1 & 0 & 1 & 3 & 3 & 0 & 4 & 2 & 3 & 3 & 24 \\
\hline
\end{tabular}

No evidence of uneven distribution throughout the year with data pooled by month (bottom row) either by $\chi^{2}$ or by the circular mean ( $p>0.1$ for both).

No evidence of uneven distribution when the years were analyzed individually (each of the first 3 rows) by $\chi^{2}(p>0.1)$ or by the circular mean $(p>0.05)$ for all years.

incidence did not vary significantly across the 3 years of the study $\left(\chi^{2}, p>0.1\right)$, with a low of 24 incidents in Year 1 (May 2005 to April 2006) and a high of 41 incidents in Year 2 (May 2006 to April 2007). Because the main focus of the study was to identify variation in incidence within the year, we pooled ISSHL incidence data across all 3 study years. As further justification for this approach, we found no significant difference in the monthly distribution of cases across each of the 3 years of the study $\left(\chi^{2}, p>0.5\right)$. Analysis of the ISSHL incidence values by month, pooled across all 3 years, yielded no evidence of uneven distribution of hearing loss throughout the year either by $\chi^{2}(p>0.1)$, which assesses for any uneven distribution, or by the circular mean $(p>0.1)$, which assesses for a pattern of seasonal variation. Additionally, no evidence was found for an uneven distribution when the years were analyzed individually by either statistical test ( $p>0.1$ for $\chi^{2}$ and circular mean, all years).

Within the whole population with greater than or equal to $20 \mathrm{~dB}$ hearing loss, 24 patients $(24.7 \%)$ reported experiencing URI symptoms before or concurrent with the onset of ISSHL (Table 2). The mean monthly incidence of hearing loss in this group was 0.67 incidents per month (range, 0-2). Monthly incidence values among this subset of patients were analyzed to identify variations throughout the year. When data from all 3 years were compiled, no evidence was found for an uneven distribution of hearing loss onset throughout the year either by $\chi^{2}$ $(p>0.1)$ or by the circular mean $(p>0.1)$. When analyzed within each year individually, this subset showed no evidence for an uneven distribution in ISSHL incidence by either statistical test ( $p>0.1$ for $\chi^{2}$ and $p>0.05$ for circular mean, all years).

Given that incidence data from the 24 patients in the URI subset did not vary significantly throughout the year, an additional analysis was performed to determine whether ISSHL incidence varied throughout the year when data from this subset of patients were excluded (i.e., patients with $\geq 20 \mathrm{~dB}$ hearing loss who did not report associated URI). Incidence data from the remaining patients (73 of 97; 75.3\%) demonstrated no evidence for an uneven distribution of hearing loss or a statistically significant peak when data were grouped by month across all 3 years of the study ( $p>0.1$ for $\chi^{2}$ and circular mean) or when monthly incidence data from each year were analyzed individually ( $p>0.1$ for $\chi^{2}$ and circular mean, all years).
When the statistical tests previously described were applied to data from the subset of patients who had experienced a hearing loss of greater than or equal to $30 \mathrm{~dB}$ (84 of 97; 86.6\%), the results were similar. No evidence was found for an uneven distribution of hearing loss or a statistically significant peak when data were grouped by month across all 3 years of the study $\left(p>0.1\right.$ for $\chi^{2}$ and circular mean) or when monthly incidence data from each year were analyzed individually $\left(p>0.05\right.$ for $\chi^{2}$ and circular mean, all years). Within this population of patients, 19 individuals $(22.6 \%)$ reported experiencing URI symptoms before or concurrent with the onset of ISSHL. Analysis of this subset of patients and the subset of patients who had not reported URI symptoms individually revealed no evidence for an uneven distribution of hearing loss or a statistically significant peak when data were grouped by month across all 3 years of the study $\left(p>0.1\right.$ for $\chi^{2}$ and circular mean) or when monthly incidence data from each year were analyzed individually $\left(p \geq 0.05\right.$ for $\chi^{2}$ and circular mean, all years).

The history data reported by the study population are presented in Table 3 . This table presents data for both the whole patient population and the subset of patients who experienced hearing loss of greater than or equal to $30 \mathrm{~dB}$. Among history data evaluated uniformly in all patients, the most frequently reported symptoms were the new onset of tinnitus ( 72 of $97 ; 74.2 \%$ ), dizziness ( 35 of $97 ; 36.1 \%$ ), aural pressure or fullness (22 of $97 ; 22.7 \%$ ), and otalgia (19 of $97 ; 19.6 \%$ ). Among history data that were not evaluated uniformly in all charts, a recent or

TABLE 3. History data evaluated in all patients with ISSHL

\begin{tabular}{lcc}
\hline & $\geq 20 \mathrm{~dB}$ & $\geq 30 \mathrm{~dB}$ \\
\hline Total & $97, \mathrm{n}(\%)$ & $84, \mathrm{n}(\%)$ \\
Tinnitus & $72(74.2)$ & $65(77.4)$ \\
Dizziness & $35(36.1)$ & $33(39.3)$ \\
Aural pressure/fullness & $22(22.7)$ & $17(20.2)$ \\
Otalgia & $19(19.6)$ & $19(22.6)$ \\
History of hearing loss & $9(9.3)$ & $9(10.7)$ \\
Familial hearing loss & $7(7.2)$ & $6(7.1)$ \\
History of noise exposure & $2(2.1)$ & $1(1.2)$ \\
\hline
\end{tabular}

All symptoms were associated with the onset of hearing loss. Data are presented separately for the group with hearing loss greater than or equal to $20 \mathrm{~dB}(\mathrm{n}=97)$ and the group with hearing loss greater than or equal to $30 \mathrm{~dB}(\mathrm{n}=84)$. 
concurrent URI was reported most often ( 24 of $97 ; 24.7 \%$ ), and headache $(22$ of $97 ; 22.7 \%$ ) was the second most frequently reported. No other pertinent history was reported in more than $10 \%$ of the population.

\section{DISCUSSION}

A number of studies have attempted to correlate viral infection with ISSHL by analyzing seasonality of symptom onset. The following includes a review of this literature.

In 2006, Wu et al. (8) conducted a retrospective crosssectional study based on national health insurance data that recorded all hospital admissions in Taiwan. The authors found that between 1998 and 2002, there were 9,267 first-time hospitalizations for the diagnosis of sudden sensorineural hearing loss (International Classification of Diseases-9th Revision-Clinical Modification code 388.2). From these data, the authors determined monthly incidence rates throughout the 5-year period. The authors then grouped these monthly values into 3month seasons and identified a statistically significant $(p<0.001)$ trend in seasonal variation, with the highest incidence rates occurring in autumn (August to October in Taiwan). In a separate study from Taiwan published in 2005, Chang et al. (9) conducted a retrospective case study of 146 patients hospitalized for ISSHL between 1997 and 2001 at a single institution. The authors reported a trend in the monthly incidence of the disease, with peaks occurring in April (15\%) and October (12\%). The authors note that in Taiwan, April and October mark transition periods between winter and spring and between summer and autumn, respectively.

In 2002, Danielides et al. (10) reviewed the case histories of 82 patients treated for ISSHL between 1995 and 1999 at a single institution in Ioannina, Greece. Patients whose histories suggested a definable cause of hearing loss were excluded. The authors identified the date on which each patient's hearing loss first occurred and used these time points to test for the relationship of the season with the incidence of disease. The authors grouped the time points within seasonal date ranges and calculated a 5 -year mean number of cases per season. The authors then tested for variation in the incidence of disease per season and found no significant differences at a $95 \%$ confidence level.

The results of the current study suggest that the incidence of ISSHL does not vary significantly throughout the year in the patient population as a whole. This finding is supported by the results presented by Danielides et al., but a seasonal incidence pattern was identified in the analysis performed by both $\mathrm{Wu}$ et al. and Chang et al.

An important distinction in comparing these studies concerns the criteria by which ISSHL was diagnosed. The NIDCD definition of ISSHL, which requires a hearing loss of greater than or equal to $30 \mathrm{~dB}$ in 3 contiguous frequencies within 3 days (4), was applied by both Chang et al. and Danielides et al., as well as in a 1983 study by Wilson et al. (11). In the study by Wu et al., no specific audiometric criteria were identified; the authors instead relied on hospitalizations with a diagnosis code of 388.2 as a measure of incidence. It is not possible to determine how many patients included in this study would have fit the NIDCD criteria for ISSHL or whether hearing loss in every patient was indeed idiopathic. As discussed previously in the Materials and Methods section, the current study included patient charts based on a hearing loss of greater than or equal to $20 \mathrm{~dB}$ from a baseline of $0 \mathrm{~dB}$ averaged over 3 contiguous audiometric frequencies, within 3 or fewer days, but also on the standard definition involving a greater than or equal to $30 \mathrm{~dB}$ threshold for hearing loss. The threshold of hearing loss greater than or equal to $20 \mathrm{~dB}$ has been used previously in a number of studies (12) and has been recommended as an alternative to the NIDCD definition because it is more sensitive in identifying patients with ISSHL (3). However, the wide usage of the NIDCD definition enables reliable comparisons between studies. The findings in the current study did not change significantly when the NIDCD criteria were applied, suggesting that these results may be compared with other studies in which the NIDCD definition was used.

Another possible explanation for the differences in the results of the current study and that of the studies previously discussed may be related to geographic location of the different patient populations. As stated previously, there are a number of hypotheses that have been proposed to explain ISSHL, and it is possible that different etiologic factors predominate in different geographic regions, leading to differences in seasonal patterns of presentation or lack thereof.

In addition, it is important to note that the current study analyzed monthly incidence values, whereas data were grouped into 3-month seasons in the study by Danielides et al. The fact that a previous study evaluated incidence in seasonal date ranges does not prevent a comparison with the current study because the circular mean test that is used in the current study enables the identification of seasonal trends in monthly incidence data. In fact, the circular mean test is a more powerful test because it does not require partitioning the year into "seasons" and thus avoids an arbitrary processing step. The advantages of the circular mean are discussed more fully in the statistical literature $(5,7,13)$.

In comparing the current study with that of $\mathrm{Wu}$ et al., it is also important to note that the number of patients studied by $\mathrm{Wu}$ et al. was significantly larger than that in the current study, and it is possible that the larger study detected small seasonal variations that would be missed in the current analysis.

A number of investigators have attempted to determine the role of viral infection in ISSHL by testing for signs of viral exposure in patients with sudden hearing loss. The findings from these studies are a subject of ongoing debate (2) and are not the focus of this discussion. However, a study from 1983 by Wilson et al. (11) in which ISSHL patients were tested for viral exposure also reported a seasonal trend in the incidence of ISSHL. 
The authors conducted a prospective cohort study that compared the incidence of viral seroconversion in an experimental group of patients with ISSHL and a control group of healthy volunteers. Patients with sudden hearing loss were evaluated to rule out definable causes of the condition. The experimental group included 122 patients seen within 10 days of the onset of ISSHL between July 1976 and January 1982 at a single institution in Boston, MA. The control group consisted of 95 volunteers recruited during the winter and spring between 1980 and 1981. The authors identified 5 viruses for which the number of positive seroconversions was found to be significantly higher among ISSHL patients than volunteers: influenza B $(p<0.001)$, mumps $(p<0.05)$, rubeola $(p<0.05)$, cytomegalovirus $(p<0.001)$, and varicella-zoster virus $(p<0.05)$. Additionally, the authors found that among subjects with ISSHL, the trend in both the incidence of seroconversions and the incidence of ISSHL followed a similar seasonal pattern, peaking in April, May, and June (the spring season in Boston); however, the authors did not perform statistical analyses to determine the significance of the trends that they observed.

Wilson et al. published the monthly ISSHL incidence data for their patient population, and it was possible to analyze these data by applying the $\chi^{2}$ and circular mean tests used in the current study. When the statistical analyses of the current study were applied to the data from Wilson et al., ISSHL incidence values were found to vary significantly throughout the year, exhibiting an uneven distribution $\left(\chi^{2}, p<0.001\right)$ and a statistically significant peak at the end of April (circular mean, $p<0.001$ ). The finding of seasonal variation in a population of patients with ISSHL known to have experienced viral infection supports the methodological approach of the current study despite the fact that the current study found no seasonal variation in a population of patients with ISSHL who reported preceding or concurrent URI symptoms. This difference may be attributed to the fact that in the current study, patient data were included in the URI subset only if the patients' charts indicated the symptoms of a URI. It is therefore unclear whether the patients included in the URI subset constitute all of the patients within the study population who experienced a URI. A prospective study in which standard history includes the presence or lack of viral exposure and viral symptoms may be warranted.

\section{CONCLUSION}

The results of this study suggest that ISSHL incidence does not display uneven distribution throughout the year.

Acknowledgments: The authors thank Ashutosh Kacker, M.D., David Kutler, M.D., Jerry Lin, M.D., Mukesh Prasad, M.D., William Reisacher, M.D., William Schley, M.D., Michael Stewart, M.D., Lucian Sulica, M.D., and Erich Voigt, M.D., for providing the patient data used in this study, and L. Teresa Valderrama, M.P.H., for coordinating the study and helping record the data.

\section{REFERENCES}

1. Byl FM Jr. Sudden hearing loss: eight years' experience and suggested prognostic table. Laryngoscope 1984;94:647-61.

2. Merchant SN, Durand ML, Adams JC. Sudden deafness: is it viral? ORL J Otorhinolaryngol Relat Spec 2008;70:52-60.

3. Hughes GB, Freedman MA, Haberkamp TJ, Guay ME. Sudden sensorineural hearing loss. Otolaryngol Clin North Am 1996;29: 393-405.

4. National Institute on Deafness and Other Communication Disorders. Sudden deafness. Available at: http://www.nidcd.nih.gov/ health/hearing/sudden.asp. Accessed May 25, 2009.

5. Mardia KV, Jupp PE. Directional Statistics. New York, NY: John Wiley \& Sons, Ltd, 2000:94-118.

6. Bradley DR, Bradley TD, McGrath SG, Cutcomb SD. Type I error rate of the chi square test of independence in $\mathrm{r} \times \mathrm{c}$ tables that have small expected frequencies. Psychol Bull 1979;86:1200-97.

7. Fisher NI. Statistical Analysis of Circular Data. Cambridge, UK: Cambridge University Press, 1993.

8. Wu CS, Lin HC, Chao PZ. Sudden sensorineural hearing loss: evidence from Taiwan. Audiol Neurotol 2006;11:151-6.

9. Chang NC, Ho KY, Kuo WR. Audiometric patterns and prognosis in sudden sensorineural hearing loss in southern Taiwan. Otolaryngol Head Neck Surg 2005;133:916-22.

10. Danielides V, Nousia CS, Bartzokas A, Lolis CJ, Kateri M, Skevas A. Weather conditions and sudden sensorineural hearing loss. BMC Ear Nose Throat Disord 2002;2:2.

11. Wilson WR, Veltri RW, Laird N, Sprinkle PM. Viral and epidemiological studies of idiopathic sudden hearing loss. Otolaryngol Head Neck Surg 1983;91:653-8.

12. O'Malley MR, Haynes DS. Sudden hearing loss. Otolaryngol Clin North Am 2008;41:633-49.

13. Brockman DK, van Schaik CP. Seasonality in Primates: Studies of Living and Extinct Human and Non-Human Primates. Cambridge, UK: Cambridge University Press, 2005:309. 